\title{
Fusarium spp en trigo, capacidad toxicogenica y quimiotaxonomia de las cepas aisladas en la Argentina
}

\author{
GA Lori 1, MR Carranza 1, A Violante 2, I Rizzo 2, HE Alippi 1 \\ 1 UNLP y Comisión de investigaciones cientificas de la provincia de Buenos Aires, \\ facultad de ciencias agrarias y forestales, cátedra de fitopatología, 60 y 118, CC 31 (1900) La Plata; \\ ${ }_{2}^{2}$ Mrio de Salud y Acción Social, Instituto nacional de farmacología y bromatología, Av Caseros (2164) Buenos Aires, Argentina
}

(Recibido el 10 de abril de 1991; aceptado el 13 de abril de 1992)

\begin{abstract}
Resumen - Se aislaron e identificaron 91 cepas de Fusarium spp ( $F$ graminearum, $F$ equiseti, $F$ moniliforme, $F m$ var anthophilum, $F$ acuminatum, $F$ solani y $F$ oxisporum) a partir de semillas de trigo pan procedentes de 17 localidades trigueras de la República Argentina. Mediante el cultivo de las mismas en arroz pelado y pulido se evaluó la producción de tricotecenos y zearalenona, a los efectos de revelar si existen diferencias regionales en cuanto a su presencia. Se detectaron cepas productoras de tricotecenos del grupo B y zearalenona, no habiéndose hallado productoras del grupo A. De las cepas evaluadas el $82,4 \%$ produjeron tricotecenos que se subdividieron en dos grupos: a) las cepas productoras de deoxinivalenol (DON) y su precursor 3 acetil-deoxinivalenol (AcDON) y b) las productoras de nivalenol (NIV) y su precursor 4 acetil-nivalenol o fusarenona-X (FUS-X). Los resultados demostraron que el $48,3 \%$ de las cepas fueron productoras de DON, el $7,7 \%$ de NIV y el $26,4 \%$ tuvieron la capacidad de producir simultaneamente DON y NIV en los cultivos. Al considerar exclusivamente las cepas de F graminearum el $89,4 \%$ fueron toxicogénicas, de ellas el $55,9 \%$ pertenecieron al quimiotipo DON, 10,3\% al quimiotipo NIV y $33,8 \%$ produjeron DON y NIV. En cuanto a la zearalenona (ZEA) el $63,2 \%$ del total de las cepas fueron productoras de esta toxina, no existiendo ninguna relación entre los grupos DON y NIV con la producción de ZEA. La distribución de las cepas toxicogénicas de Fusarium spp observada en las distintas localidades de la República Argentina, permite inferir la existencia de diferencias regionales en el área triguera.
\end{abstract}

\section{Fusarium spp / trigo / tricoteceno / zearalenona / quimiotipo / Argentina}

Summary - Fusarium spp in wheat, toxicogenic capacity and chemotaxonomy of the strains isolated in Argentina. In wheat seeds from 17 different wheat growing sites, 91 Fusarium spp strains ( $F$ graminearum, $F$ equiseti, $F$ moniliforme, $F m$ var anthophilum, $F$ acuminatum, $F$ solani and $F$ oxysporum) were isolated and identified. The production of trichothecenes and zearalenone was evaluated after growing them on peeled and polished rice to find out if there were any regional differences in their prevalence. Strains producing trichothecenes of the B group and zearalenone were detected; no producers of the A groups were found. From the strains studied, $82.4 \%$ produced trichothecenes, and could be divided into 2 groups: a), deoxynivalenol (DON) producing strains and its precursor 3 acetyldeoxynivalenol (AcDON); and b), nivalenol (NIV) producing strains and its precursor 4 acetylnivalenol or fusarenone-x (FUS-X). The results showed that $48.3 \%$ of the strains were DON producers, $7.7 \%$ NIV producers and $26.4 \%$ produced both DON and NIV. Eighty-nine point four percent $F$ graminearum strains were toxigenic; among them $55.9 \%$ were DON chemotype, $10.3 \%$ NIV chemotype and $33.8 \%$ produced DON and NIV. Sixty-three point two percent of the strains also produced zearalenone (ZEA), no relation existing between the DON and NIV groups with regard to ZEA production. The finding of certain toxigenic strains in various sites in Argentina allows us to conclude that regional differences exist.

Fusarium spp / wheat / trichothecenes / zearalenone / chemotype / Argentina 


\section{INTRODUCTION}

Fusarium graminearum Schwabe, en la República Argentina como en el resto del mundo, es la especie del género Fusarium más frecuente en trigo (Carranza, 1961; Tuite et al, 1974; Ichinoe et al, 1983). Ocasionalmente aparecen otras especies que junto a la mencionada son productoras de micotoxinas tales como zearalenona y sus derivados, responsable de un síndrome estrogénico (Stob et al, 1962) y tricotecenos. Estos últimos han sido separados en dos categorías: Grupo A, constituído por la toxina T2; T-2 tetraol; neosolaniol; diacetoxiscirpenol (DAS) y acetil T-2 como los más tóxicos. Todos estos son altamente peligrosos pues producen irritación dérmica, náuseas, vómitos, diarreas, abortos, alteraciones hematológicas (leucopenia), actúan como carcinogénicos y pueden llegar a ocasionar la muerte tanto en el hombre como en otros animales. El Grupo B, constituido por deoxinivalenol (DON), nivalenol (NIV) y fusarenona-X (FUS-X), como las más importantes, originan alteraciones digestivas sin liegar a producir la muerte; pero de hallarse juntos DON y NIV la toxicidad se acentúa (Yoshizawa y Morooka, 1974; Ueno, 1983).

Estudios recientes revelaron que NIV y DON están presentes como toxinas contaminantes en muestras de cereales procedentes de distintas partes del mundo. Se han encontrado diferencias regionales en cuanto a la ocurrencia de estos tricotecenos. Así en USA (Vesonder et al, 1978), Canadá (Scott et al, 1982) y Sudáfrica (Marasas et al, 1979) DON es el tricoteceno más común en trigo. Mientras que en Japón (Kuroda et al, 1979) y en Francia (Jemmali et al, 1978) tanto NIV como DON se presentan indistintamente en los granos contaminados.

Ichinoe et al (1983) estudiaron en Japón la existencia de quimiotipos de Gibberella zeae (Schw) Petch (Fusarium graminearum) y así detectaron diferencias regionales en la distribución de los mismos.

Con referencia a la «fusariosis» o «golpe blanco" del trigo, cabe señalar que en la República Argentina durante las dos últimas décadas se ha constituído en una de las principales patologías del cultivo (Carranza y Arriaga, 1988), dependiendo los niveles de infección alcanzados en las diferentes campañas de las condiciones climáticas y de la época de siembra. Durante el período 85-86 la contaminación con F graminearum fue sumamente elevada (Carran- za y Arriaga, 1988) y, consecuencia de ello, se encontraron alimentos elaborados con harina de trigo contaminados con tricotecenos, por ejemplo: DON, NIV, toxina T-2, neosolaniol y acetil T2 (Rizzo, com pers). Sin embargo al analizarse la capacidad toxicogénica de 24 aislamientos de $F$ graminearum, que procedieron de varias localidades de dos provincias de la Argentina, se detectó que sólo el $33 \%$ de los aislamientos fueron productores de toxinas y entre los tricotecenos sintetizados predominó el DON, siendo los resultados negativos para el NIV o su precursor FUSX (Faifer et al, 1990).

Es de destacar que en el país la superficie sembrada con el cultivo de trigo es sumamente amplia y distribuída en cinco provincias, que al poseer diferentes condiciones agrometeorológicas (fig 1) podrían condicionar la aparición de cepas de Fusarium spp productoras de diferentes toxinas. El objetivo de este trabajo consistió en determinar la existencia de dichas cepas en el área triguera de la República Argentina.

\section{MATERIALES Y METODOS}

En la República Argentina la región triguera abarca une superficie aproximada de 4660000 ha distribuída en 5 provincias (Buenos Aires, Santa Fe, Córdoba, La Pampa y Entre Ríos). De acuerdo con las condiciones agrometeorológicas existentes (fig 1), a consecuencia de la gran extensión, la Red Oficial de Ensayos Territoriales (ROET) establece de 2 a 6 épocas de siembra entre los meses de mayo y agosto, recomendando para cada una correspondientes variedades que varían de 10 a 30 según la localidad y época de siembra.

\section{Origen de las muestras}

Las semillas de trigo pan correspondientes a la campaña 87-88 fueron remitidas por la Junta Nacional de Granos. Se analizaron 405 muestras que procedieron de 17 localidades de cultivo ubicadas en las provincias de Buenos Aires (Barrow, Pergamino, Miramar, Balcarce, Carhué, Bordenave, La Dulce, Plá y Coronel Suárez), Entre Ríos (Paraná), La Pampa (Anguil y General Pico), Córdoba (Marcos Juárez y Villa del Rosario) y Santa Fe (Oliveros, Rafaela y Reconquista) (fig 1).

\section{Análisis de sanidad}

Se aplicaron las normas ISTA (Neergaard, 1974) para el método del papel de filtro. Cada una de las mues- 


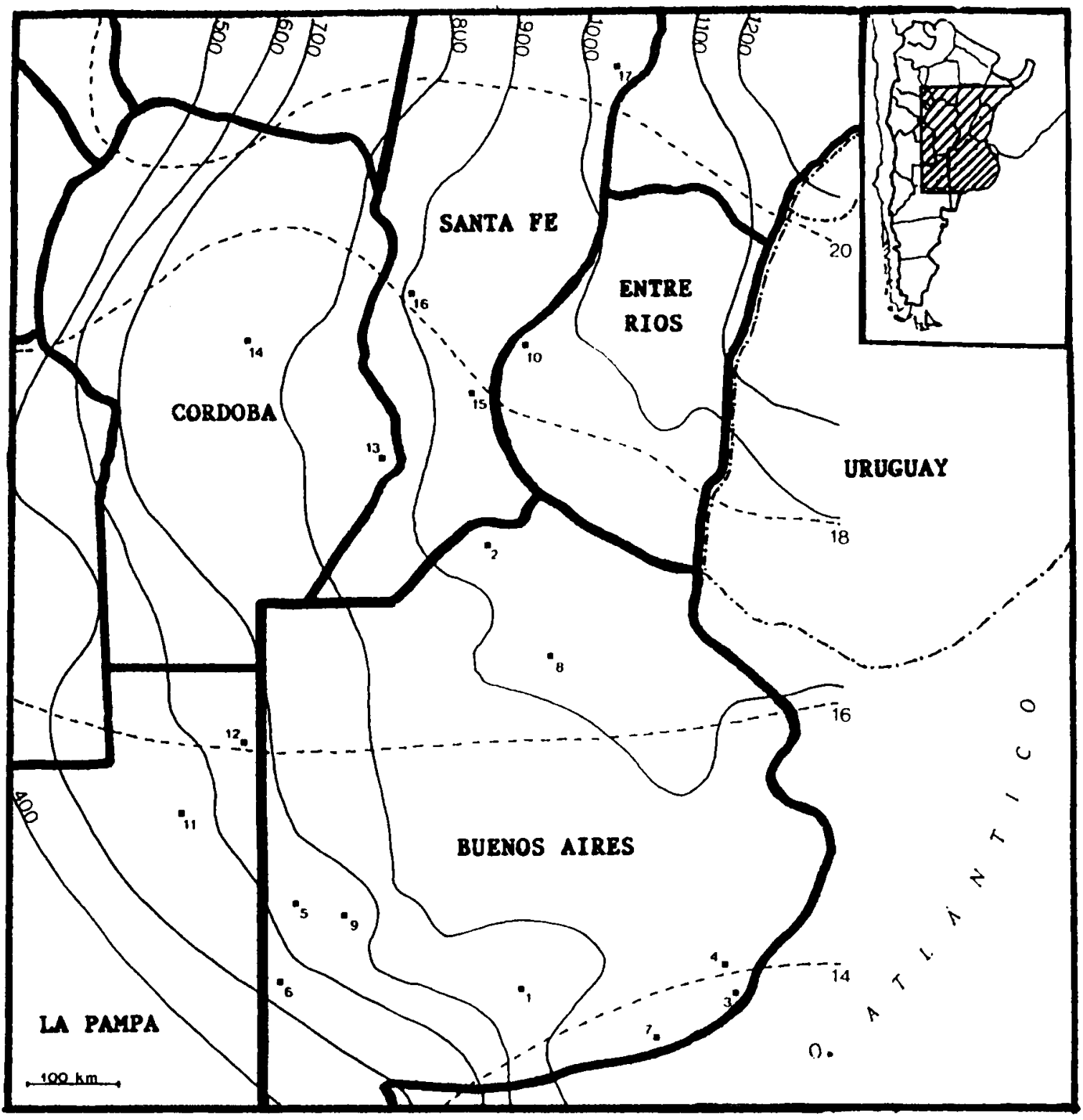

Fig 1. Area triguera de la República Argentina. Localidades analizadas: 1. Barrow; 2. Pergamino; 3. Miramar; 4. Balcarce; 5. Carhué; 6. Bordenave; 7. La Dulce; 8. Plá; 9. Coronel Suárez (Provincia de Buenos Aires); 10. Paraná (Provinica de Entre Ríos); 11. Anguil; 12. General Pico (Provincia de La Pampa); 13. Marcos Juárez; 14. Villa del Rosario (Provincia de Córdoba); 15. Oliveros; 16. Rafaela; 17. Reconquista (Provincia de Santa Fe).

Limite de provincias. ------ Temperatura media anual $\left({ }^{\circ} \mathrm{C}\right) . \_$Precipitación media anual (mm).

tras estuvo constituída por 400 semillas. En aquellas que evidenciaron la presencia de micelio $y / 0$ pionótides correspondientes a Fusarium spp se practicó un aislamiento directo. La identificación de las especies se llevó a cabo mediante el estudio de sus caracteres morfobiométricos y culturales de acuerdo al sistema de clasificación de Booth (1971).

Para determinar la capacidad toxicogénica se seleccionaron aislamientos de $F$ graminearum (especie más frecuente en trigo) procedentes de la época que manifestó mayor registro de infección en cada localidad analizada. También se evaluó la capacidad toxico- génica de las restantes especies cuya aparación resultó esporádica.

\section{Evaluación de la capacidad toxicogénica}

\section{Preparación de las muestras}

Las cepas seleccionadas e identificadas se cultivaron en erlenmeyers de $500 \mathrm{ml}$ utilizando como sustrato $50 \mathrm{~g}$ de arroz pelado y pulido. La hidratación de estos 
últimos se logró por inmersión en agua corriente durante $1 \mathrm{~h}$. Los granos así tratados se escurrieron y esterilizaron en autoclave a $120^{\circ} \mathrm{C}$ durante $20 \mathrm{~min}$. Se adicionaron $5 \mathrm{ml}$ de una solución estéril de peptona al $10 \%$, se sembraron con las cepas seleccionadas provenientes de cultivos monospóricos y se incubaron a $25^{\circ} \mathrm{C}$ en cámara de cultivo durante 20 días. Finalizado el período de incubación las muestras se secaron en estufa con circulación de aire a $70^{\circ} \mathrm{C}$ durante $24 \mathrm{~h}$ y se molieron con molinillo para grano.

\section{Extracción y detección de micotoxinas}

Se realizó según la técnica de Bottalico et al (1983). Como solvente para la extracción se utilizó metanolagua (40:60) más una solución de cloruro de sodio al $2 \%$. La mezcla constituída por la muestra molida y el solvente se agitó durante $30 \mathrm{~min}$ en agitador mecánico. Se filtró a través de papel de filtro de gran porosidad y la solución obtenida se trató con hexano en una ampolla de decantación. La porción metanólica se llevó a sequedad en evaporador rotatorio, se agregaron $3 \mathrm{ml}$ de acetato de etilo en baño de maría para redisolver el extracto y aumentar la solubilidad de las toxinas, que de otra manera quedarian adheridas a las paredes del balón. Se transvasó a otro balón y se llevó nuevamente a sequedad en evaporador rotatorio y el extracto se redisolvió en $100 \mu \mathrm{l}$ de cloroformo. Para la detección de las distintas toxinas, los extractos obtenidos se sembraron en placas de sílica gel 60, sin indicador de fluorescencia, junto con los standards cualitativos de las micotoxinas (DON; NIV; AcDON; FUS-X; ZEA; zearalenol; Toxina T-2 y DAS). El solvente de desarrollo usado fue bencenoacetona (3:2). Los sistemas de revelado fueron para el grupo $B$ cloruro de aluminio al $15 \%$ en agua y etanol $(15: 85)$ y para el grupo A ácido sulfúrico al $25 \%$ (Scott, 1981). En el caso de ZEA y zearalenol se utilizaron volúmenes iguales de una solución de benzidina al $0,5 \%$ en ácido clorhídrico y agua $(1,5: 98,5)$ y solución de nitrato de sodio al $10 \%$ (Schulter y van Egmond, 1983).

\section{RESULTADOS}

\section{Análisis de sanidad}

Los porcentajes de infección alcanzados fueron muy variables, dependiendo tanto de la procedencia de la muestra como de su época de siembra. En cuanto a la primera, se pudo apreciar que en localidades marginales como $\mathrm{Pa}$ raná, Reconquista o Villa del Rosario los registros alcanzaron hasta un $40 \%$, mientras que en el resto de las localidades pertenecientes a la región triguera por excelencia, provincia de Buenos Aires, sur de Santa Fe y sudeste de
Córdoba (fig 1), no superaron el $12 \%$. Semejante comportamiento se observó en las épocas de siembra más tardías (tabla l).

En las 405 muestras analizadas, $F$ graminearum fue la especie predominante, más del $90 \%$, las restantes aparecieron en forma esporádica (tabla I). Se seleccionaron 91 cepas distribuídas de la siguiente manera: $F$ graminearum 76 cepas; $F$ equiseti 7 cepas; $F$ moniliforme 3 cepas; $F m$ var anthophilum 1 cepa; $F$ solani 1 cepa; $F$ oxysporum 1 cepa y $F$ acuminatum 2 cepas.

\section{Capacidad toxicogénica}

Bajo las condiciones de cultivo empleadas, los resultados revelaron el predominio de cepas capaces de elaborar tricotecenos del grupo $B$, ZEA y zearalenol, mientras que no se detectaron cepas productoras de tricotecenos del grupo A, T-2 y DAS.

De las 91 cepas analizadas, $75(82,4 \%)$ se comportaron como toxicogénicas. Cabe resaltar que las 16 cepas restantes $(17,6 \%)$ que no produjeron toxinas fueron fundamentalmente aquellas especies cuya aparición en trigo resulta esporádica ( $F$ solani, $F$ oxysporum, etc) (tabla I).

Considerando los tricotecenos sentetizados, las cepas toxicogénicas se trataron de agrupar en dos tipos o grupos según Ichinoe et al (1983): - las productoras de DON y su precursor 3 acetil-deoxinivalenol (Ac DON) y

- las productoras de NIV y su precursor 4 acetilnivalenol o fusarenona-X (FUS-X).

En las distintas localidades estudiadas se detectaron 44 cepas $(48,3 \%)$ con capacidad de producir DON, $7(7,7 \%)$ productoras de NIV y un grupo de 24 cepas $(26,4 \%)$ metabolizaron en sus cultivos DON y NIV simultaneamente (tabla 1).

Al considerar exclusivamente el comportamiento de las cepas de $F$ graminearum, de las 76 analizadas $(83,5 \%$ del total), $68(89,4 \%)$ manifestaron poseer capacidad toxicogénica, de ellas $38(55,9 \%)$ fueron del tipo DON, $7(10,3 \%)$ del tipo NIV y $23(33,8 \%)$ produjeron DON y NIV. En todas las localidades analizadas se detectaron cepas de $F$ graminearum toxicogénicas (tabla II).

En cuanto a la producción de ZEA, 48 cepas de $F$ graminearum $(63,2 \%$ del total) manifestaron esta capacidad. No se halló ninguna relación entre los quimiotipos DON y NIV y la producción de 
Tabla I. Cepas de Fusarium spp aisladas de semillas de trigo pan procedentes de distintas localidades y épocas de siembra. Micotoxinas producidas por ellas.

(a) Provincia

(b) Localidad y época de siembra con mayor registro de infección.

(c) Cepas de Fusarium spp aisladas, F gram: Fusarium graminearum; F oxys: $F$ oxysporum; $F$ equi: $F$ equiseti; $\mathrm{F}$ moni: $F$ moniliforme; $\mathrm{F}$ moni v anth: $F$ moniliforme var anthophilum; $\mathrm{F}$ sol: $F$ solani; $\mathrm{F}$ acum: Fusarium acuminatum.

(d) Tricotecenos, DON: deoxinivalenol; ACDON: acetil deoxinivalenol; NIV: nivalenol; FUS-X: fusarenona-X.

(e) Zearalenona, ZEA: zearalenona: ZEANOL: zearalenol.

* Toxina detectada

- Toxina no detectada

(f) 4ta época de siembra, 1.7.87; 5ta época de siembra, 20.7.87; 6ta época de siembra, 10.8.87
(a)
(b)
Cepas (c)
$D O N \quad A C D O N$
(d)
(e)

Buenos Aires

Barrow 5ta época ( $(t)$

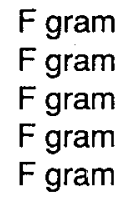

Pergamino 5ta época

$\begin{array}{lllllll}\text { F gram } & * & * & - & - & * & - \\ \text { F gram } & * & - & - & - & * & - \\ \text { F gram } & * & - & - & - & * \\ \text { F gram } & * & - & - & - & * \\ \text { F gram } & * & - & - & - & * & - \\ \text { F oxys } & - & - & - & - & - & -\end{array}$

Miramar 4ta-5ta época

F gram

F gram

F gram

$F$ gram

$F$ gram

F gram

Balcarce 5ta época

F gram

F gram

$F$ gram

$F$ gram

$F$ gram

Carhué 5ta época

$$
\begin{aligned}
& F \text { gram } \\
& F \text { gram } \\
& F \text { equi } \\
& F \text { equi } \\
& F \text { equi }
\end{aligned}
$$

Bordenave 5ta época

$\begin{array}{lllllll}\text { Fgram } & * & - & - & - & * & - \\ \text { Fgram } & * & - & - & - & * & - \\ \text { F moni } & * & * & - & - & - & - \\ \text { F moni } & * & - & - & - & * & - \\ \text { F moni } & * & - & - & - & * & -\end{array}$


(a)

(b)

Cepas (c)

(d)

$D O N \quad A C D O N$

NIV

FUS-X

(e)

ZEA ZEANOL

La Dulce 5ta época

$F$ gram

$F$ gram

$\mathrm{F}$ gram

F gram

F gram

Plá 5ta época

F gram

F gram

$F$ gram

$F$ gram

$F$ gram

Coronel Suárez 5ta época

F gram

$F$ gram

$F$ gram

$F$ gram

$F$ gram

$F$ equi

Entre Ríos

Paraná 6ta época

$\begin{array}{lllllll}\text { F gram } & * & - & - & - & - & - \\ \text { F gram } & * & * & * & - & * & * \\ \text { F gram } & * & - & - & - & * & - \\ \text { F gram } & - & - & * & * & * & * \\ \text { F gram } & * & - & * & * & - & -\end{array}$

La Pampa

Anguil 4ta época

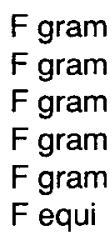

General Pico 4ta época

F gram

$F$ gram

$F$ gram

$F$ gram

$F$ equi

Cordoba

Marcos Juaréz 4ta época

$$
\begin{aligned}
& \text { F gram } \\
& F \text { gram } \\
& F \text { gram } \\
& \text { F gram } \\
& \text { F gram }
\end{aligned}
$$



(a)
(b)
Cepas (c)
DON ACDON NIV FUS-X
(e)
ZEA ZEANOL

(d)

Villa del Rosario 4ta época

$F$ gram
$F$ gram
$F$ gram
$F$ gram
$F$ sol
$F$ moni
$V$ anth

Santa Fe

Oliveros 5ta época

$$
\begin{aligned}
& F \text { gram } \\
& F \text { gram } \\
& F \text { gram } \\
& F \text { acum } \\
& F \text { acum } \\
& F \text { equi }
\end{aligned}
$$

Rafaela 6ta época

$$
\begin{aligned}
& F \text { gram } \\
& F \text { gram } \\
& F \text { gram } \\
& F \text { gram } \\
& F \text { gram }
\end{aligned}
$$

Reconquista 4ta época

$$
\begin{aligned}
& F \text { gram } \\
& F \text { gram } \\
& F \text { gram } \\
& F \text { gram } \\
& F \text { gram }
\end{aligned}
$$

ZEA. La elaboración fue indistinta para ambos tipos de cepas.

Aunque el mayor número de cepas toxicogénicas correspondió a $F$ graminearum, también se detectaron productoras de tricotecenos del grupo $B$ entre las cepas de $F$ equiseti y $F$ moniliforme (tabla I).

\section{DISCUSION}

Los resultados obtenidos demuestran que en la República Argentina, al igual que en otras partes del mundo (Neish y Cohen, 1981; Yoshizawa y Hosokawa, 1983) las cepas de Fusarium spp aisladas de trigo y fundamentalmente $F$ graminearum son toxicogénicas y que el mayor número de ellas producen DON. Por su parte Ichinoe et al (1983), al analizar las cepas de Gibberella zeae y $F$ graminearum obtenidas de distintos cereales en Japón, hallaron un marcado predominio del quimiotipo NIV en las cepas aisladas de trigo y cebada, mientras que a diferencia de lo detectado en Argentina no encontraron ningún quimiotipo DON entre los aislamientos de trigo. También en contraste con dicha investigación algunas de las cepas seleccionadas en este trabajo no pertenecieron a un quimiotipo $u$ otro, sino que sintetizaron ambas toxinas (DON y NIV), lo que deja planteado un interrogante a resolver en futuros estudios, ya que si se lograra confirmar estos resultados mediante una metodología más rigurosa, podríamos afirmar que en Argentina estamos en presencia de un nuevo quimiotipo, tal como fue demostrado por Sugiura et al (1990). 
Tabla II. Producción de tricotecenos y zearalenona por cepas de Fusarium graminearum aisladas de semillas de trigo pan procedentes de distintas localidades de la República Argentina. (a) Número de cepas en las cuales se detectó tricotecenos sobre el número de cepas evaluadas (DON; AcDON; NIV y FUS-X); (b) Número de cepas en las cuales se detectó zearalenona sobre el número de cepas evaluadas.

\section{Provincia Localidad Tricotecenos Zearalenona}

(a)

(b)

\begin{tabular}{|c|c|c|c|}
\hline \multirow[t]{9}{*}{ Buenos Aires } & Barrow & $5 / 5$ & $1 / 5$ \\
\hline & Pergamino & $5 / 5$ & $5 / 5$ \\
\hline & Miramar & $6 / 6$ & $5 / 6$ \\
\hline & Balcarce & $4 / 5$ & $2 / 5$ \\
\hline & Carhué & $2 / 2$ & $0 / 2$ \\
\hline & Bordenave & $2 / 2$ & $2 / 2$ \\
\hline & La Dulce & $4 / 5$ & $4 / 5$ \\
\hline & Plá & $4 / 5$ & $4 / 5$ \\
\hline & $\begin{array}{l}\text { Coronel } \\
\text { Suárez }\end{array}$ & $3 / 5$ & $3 / 5$ \\
\hline Entre Ríos & Paraná & $5 / 5$ & $3 / 5$ \\
\hline \multirow[t]{2}{*}{ La Pampa } & Anguil & $5 / 5$ & $4 / 5$ \\
\hline & $\begin{array}{l}\text { General } \\
\text { Pico }\end{array}$ & $4 / 4$ & $2 / 4$ \\
\hline \multirow[t]{2}{*}{ Córdoba } & $\begin{array}{l}\text { Marcos } \\
\text { Juárez }\end{array}$ & $4 / 5$ & $2 / 5$ \\
\hline & $\begin{array}{l}\text { Villa } \\
\text { del Rosario }\end{array}$ & $4 / 4$ & $4 / 4$ \\
\hline \multirow[t]{3}{*}{ Santa Fe } & Oliveros & $3 / 3$ & $1 / 3$ \\
\hline & Rafaela & $5 / 5$ & $5 / 5$ \\
\hline & Reconquista & $4 / 5$ & $2 / 5$ \\
\hline
\end{tabular}

En cuanto a la distríbứción de las cepas toxicogénicas de Fusariuitîn spp, se detectaron cepas productoras de DON en todas las localidades analizadas dèl área triguera argentina, sin embargo en un rúmero elevado de ellas se hallaron conjuntamente productoras de DON y NIV. La distribución geográfica no fue uniforme, se observaron diferencias regionales, pues en la provincia de Buenos Aires donde se concentra la mayor superficie (3 100000 ha) y producción triguera argentina $(5300000 \mathrm{t})$ fue relevante la presencia del quimiotipo DON entre las cepas de $F$ graminearum $(67 \%)$, mientras que en el resto de las provincias que limitan con aquella estuvieron presentes ambos quimiotipos (tabla I).
La presencia del quimiotipo NIV en la Argentina difiere de los datos de Faifer et al (1990), quienes no hallaron cepas de $F$ graminearum productoras de NIV/FUS-X entre aislamientos realizados a partir de trigo procedente de dos provincias trigueras argentinas. Los resultados del presente trabajo coincidirían con las investigaciones efectuadas en Francia, en Japón y en otros países (Jemmali et al, 1978; Kuroda et al, 1979) donde DON y NIV están presentes en cultivos infectados por $F$ graminearum.

El hallazgo de quimiotipos NIV en la Argentina debe ser tenido en cuenta sobre todo al efectuar el control de ciertos alimentos, pues pese a que DON y NIV se asemejan quimicamente, NIV es considerablemente más tóxico como fue demostrado mediante bioensayos por Yoshizawa y Morooka (1974).

La capacidad que demostraron las cepas de producir ZEA y tricotecenos en un mismo cultivo confirma que ambos grupos de toxinas pueden coexistir naturalmente (Mirocha et al, 1976; Marasas et al, 1977). Con referencia a ello y de acuerdo con Ichinoe et al (1983) la producción de ZEA no resultó exclusiva del quimiotipo DON o del quimiotipo NIV.

La detección de tricotecenos del grupo $\mathrm{B}$ en los cultivos de $F$ equiseti coincide con las investigaciones realizadas por Greenhalgh et al (1985) cuando evaluó la toxicidad potencial de aislamientos de Fusarium spp recolectados en el sudeste asiático, mientras que no se hallaron antecedentes de la producción de tricotecenos por parte de $F$ moniliforme.

Con respecto a las diferencias regionales, debido a la gran extensión del área triguera argentina que presenta marcadas variaciones de temperatura y humedad (fig 1), no se pueden extraer conclusiones tan precisas como en otros países donde la distribución de DON y NIV es uniforme. Sin embargo cabe destacar que en gran parte de nuestro territorio prevalecen las condiciones climáticas que favorecen la aparición de $F$ graminearum en trigo con capacidad de producir DON y NIV. El mayor número correspondería al quimiotipo DON.

El presente es el primer trabajo que incluye el análisis toxicogénico de cepas de Fusarium spp procedentes de toda el área triguera argentina, por lo que, de acuerdo a lo expuesto, serán necesarios futuros estudios tendientes a ampliar y profundizar estos resultados. 


\section{AGRADECIMIENTOS}

Los autores agradecen al Ing agr HO Arriaga, profesor de Cerealicultura (UNLP) y a la Junta Nacional de Granos por la remisión de las muestras analizadas. A la Sta M Haidukowski por el aporte técnico y a la profesora SA Moya por la colaboración prestada en la redacción del Summary.

El presente trabajo fue financiado parcialmente por la Comisión de Investigaciónes Científicas de la provincia de Buenos Aires y por CAFPTA.

\section{REFERENCIAS}

Booth C (1971) The Genus Fusarium. Comm Mycol Inst, Kew, England, $237 \mathrm{p}$

Bottalico A, Lerario P, Visconti A (1983) Production of mycotoxins (zearalenone, trichothecene and moniliformin) by Fusarium species in Italy. Microb Alim Nutr 1, 133-142

Carranza JM (1961) Podredumbre radical y tizón de los cereales en la República Argentina, producido por Gibberella zeae (Fusarium graminearum). Rev Fac Agron 37, 33-58

Carranza MR, Arriaga HO (1988) Control químico del tizón de la plántula en trigo. Turrialba 38, 306-308

Faifer G, Sala de Miguel M, Godoy H (1990) Patterns of mycotoxin production by Fusarium graminearum isolated from Argentine wheat. Mycopathologia $109,165-170$

Greenhalgh R, Miller JD, Neish GA, Schiefer HB (1985) Toxigenic potential of some Fusarium isolates from Southeast Asia. Appl Environ Microbiol 50, 550-552

Ichinoe M, Kurata H, Sugiura Y, Ueno Y (1983) Chemotaxonomy of Gibberella zeae with special reference to production of trichothecenes and zearalenone. App/ Environ Microbio/ 46, 1364-1369

Jemmali $M$, Ueno $Y$, Ishii $K$, Frayssinet $C$, Ethienne $M$ (1978) Natural occurrence of trichothecenes (nivalenol, deoxynivalenol, T2) and zearalenone in corn. Experientia 34, 1333

Kuroda $\mathrm{H}$, Mori T, Nishioka $\mathrm{C}$, Okasaki H, Takagi M (1979) Studies on the gas chromatographic determination of trichothecene mycotoxins in food. J Food Hyg Soc Jpn 20, 137-142

Marasas WFO, Kriek NPJ, van Rensburg SJ, Stein M, Schalkwyk GC (1977) Occurrence of zearalenone and deoxynivalenol, mycotoxins produced by $\mathrm{Fu}$ sarium graminearum Schwabe, in maize in Southern Africa. S Afr J Sci 73, 346-349
Marasas WFO, van Rensburg SJ, Mirocha CJ (1979) Incidence of Fusarium species and the mycotoxins, deoxinivalenol and zearalenone corn produced in esophageal cancer area in Transkei. J Agric Food Chem 27, 1108-1112

Mirocha CJ, Pathre SV, Schauerhamer B, Christensen CM (1976) Natural occurrence of Fusarium toxins in feed-stuff. Appl Environ Microbiol 32, 553-556

Neergaard P (1974) Report of the fourth regional workshop on seed pathology for developing countries. Danish Goverment Inst Seed Pathol for developing countries, Denmark, $22 p$

Neish GA, Cohen H (1981) Vomitoxin and zearalenone production by Fusarium graminearum from winter wheat and barley in Ontario. Can J Plant Sci $61,811-815$

Scott MP (1981) Analysis of mycotoxins other than aflatoxins in food stuffs. Workshop on Mycotoxins Analytical Methods, Egyp 17, 251

Scott MP, Kanhere S, Lau P (1982) Methodology for trichothecene. Fifth Int IUPAC Symp Mycotoxins and Phycotoxins, 44-47

Schulter PL, van Egmond HP (1983) A concise manual of thin layer chromatography laboratory technique. Workshop on Mycotoxins Analysis, Egypt $22 \mathrm{p}$

Stob M, Baldwin RS, Tuite J, Andrew FN, Gillete KG (1962) Isolation of an anabolic, uterotrophic compound of corn infected with Gibberella zeae. Nature 196, 1318

Sugiura $Y$, Watanabe $Y$, Tanaka T, Yamamoto $S$, Ueno $Y$ (1990) Occurrence of Gibberella zeae strains that produce both nivalenol and deoxinivalenol. Appl Environ Microbiol 56, 3047-3051

Tuite J, Shaner G, Rambo G, Foster J, Caldwell RW (1974) The Gibberella ear rot epidemics of corn in Indiana in 1965 and 1972. Cereal Sci Today 19, 238-241

Ueno $Y$ (1983) Toxicology of trichothecenes. In: Trichothecenes: chemical, biological and toxicological aspects. Kodansna Ltd, Jpn, 135-195

Vesonder RF, Ciegler A, Rogers RF, Brudridge KA, Bothast RJ, Jensen AH (1978) Survey of 1977 crop year preharvest corn for vomitoxin. Appl Environ Microbio/ 36, 885-888

Yoshizawa T, Morooka N (1974) Studies on the toxic substances in the infected cereals. III. Acute toxicities of new trichothecene mycotoxins: deoxynivalenol and its monoacetates. J Food Hyg Soc Jpn 15, 261-269

Yoshizawa T, Hosokawa H (1983) Natural occurrence of deoxynivalenol and nivalenol, trichothecene mycotoxins, in commercial foods. J Food Hyg Soc Jpn 24, 413-415 